\title{
Power Spectral Analysis of Orthogonal Pulse-Based TH-UWB Signals
}

\author{
Sudhan Majhi, Paul Richardson \\ Electrical and Computer Engineering, University of Michigan, Dearborn, USA \\ E-mail:sudhanmajhi@gmail.com, richarpc@umd.umich.edu \\ Received April 28, 2010; revised July 8, 2010; accepted September 10, 2010
}

\begin{abstract}
The paper analyzes power spectral density (PSD) of orthogonal pulse-based signals for time hopping ultra wideband (TH-UWB) systems. Our extensive studies show that the PSD of these signals depends not only on the time dithering code and the modulation schemes, but also on the energy spectral density (ESD) of orthogonal pulses. The different order orthogonal pulses provide different ESD which changes the shape of continuous spectral component with symbols. We show that orthogonal pulse-based signals reduce the dynamic range of amplitude of discrete spectral components. Further, we reduce the dynamic range by adopting longer TH code over orthogonal pulse-based signals. As a result, UWB system performance improves with average transmitted power. The theoretical analysis of PSD of orthogonal pulse-based TH-UWB signal is provided in details and verified through simulation results.
\end{abstract}

Keywords: Time Hopping Ultra Wideband Radio, Orthogonal Pulse Modulation, Power Spectral Density

\section{Introduction}

Impulse radio Ultra Wideband (IR-UWB) radio is a promising technology for short range wireless communications. The information is conveyed by short-duration pulse, which has potential to deliver high data rates with low power spectral densities (PSD). Federal Communication Commission (FCC) has limited the PSD of the signal to $-41.25 \mathrm{dBm} / \mathrm{MHz}$ in the band from $3.1 \mathrm{GHz}$ to 10.6 GHz. However, the UWB signal interferes with other radio signals due to its noise like signal characteristic. The power distribution of UWB signal over a large bandwidth can be identified by analyzing the PSD of the signal. The reduction of PSD of UWB signal reduces UWB interference on narrowband (NB) systems and keeps UWB signal under the FCC spectral mask [1].

Recently, orthogonal pulses have taken a lot of attention in time hopping UWB (TH-UWB) systems [2]. Several modulation schemes such as pulse shape modulation (PSM), combined on-off-keying (OOK) and PSM, called OOK-PSM, combined PSM and pulse position modulation (PPM), called PSM-PPM, combined orthogonal PPM (OPPM) and bi-orthogonal PSM (BPSM), called OPPM-BPSM, have been proposed to simplify the TH-UWB systems [3-5]. The orthogonal pulse-based modulation schemes are becoming more popular due to the simplicity of newly developed pulse designed algorithms [6,7]. The efficiency of these orthogonal pulses is studied in $[8,9]$. The spectral characteristic of these orthogonal pulses is provided in [10]. However, only few papers described the PSD of orthogonal pulse-based modulation schemes.

In this paper, we analyze the power spectral density of orthogonal pulse-based TH-UWB signal for PSM, BPSM, OPPM-PSM and OPPM-BPSM schemes. We show that orthogonal pulse-based modulation schemes adjust the continuous power spectrum component with FCC limitation and reduce the dynamic range of the amplitude of the discrete spectral components. Again we smooth the PSD of the signal by employing longer TH code over the orthogonal pulse-based modulation. The simulation results are provided for PSD of TH-UWB signal for OPPM and OPPM-PSM schemes based on modified Hermite pulses (MHPs).

The rest of the paper is organized as follows. Section 2 discusses a generalized orthogonal pulse-based TH-UWB signal. Section 3 presents derivation of PSD of orthogonal pulse-based TH-UWB signal. Section 4 characterizes PSD of TH-UWB signal for PSM, BPSM and OPPMBPSM schemes. In Section 5, simulation results and discussion are provided. Finally, conclusion is drawn in Section 6. 


\section{Generalized Orthogonal Pulse-Based UWB Signal Model}

In $M$-ary PSM scheme, different symbols are transmitted by different order orthogonal pulses. It requires $M$ orthogonal pulses at the transmitter and $M$ correlators at the receiver. In $M$-ary BPSM scheme, symbols are transmitted by using positive or negative amplitude of the orthogonal pulses. It requires $M / 2$ orthogonal pulses at the transmitter and $M / 2$ correlators at the receiver to transmit and receive all $M$ possible symbols. In OPPMBPSM scheme, $M$ symbols are transmitted by using $L$ pulse positions and $N$ biorthogonal pulses where $M=2^{k}, L=2^{l}$ and $N=2^{k-l-1}$ [5].

OPPM-BPSM is a generalized modulation scheme of PSM, BPSM, OPPM-PSM and OPPM-PSM scheme [5]. By changing the number of pulse positions and orthogonal pulses, one can construct a wide variety of modulaitons. For example, $M$-ary PSM scheme can be designed by using one pulse position and $M$ orthogonal pulses whereas an $M$-ary BPSM can be constructed by using one pulse position and $M / 2$ biorthogonal pulses. $M$-ary OPPM-PSM can be constructed by using $L$ positions and $2 N$ orthogonal pulses.

Since PSM, BPSM and OPPM-PSM are particular cases of OPPM-BPSM, only OPPM-BPSM signal model is provided. The $M$-ary OPPM-BPSM signal for $k$ th user is given as

$$
s_{m n l}^{(k)}(t)=\sum_{j} \sqrt{E_{t x}} d_{m} w_{n}^{(k)}\left(t-j T_{f}-c_{j}^{(k)} T_{c}-\delta_{l}^{(k)}\right)
$$

where $j$ is the index of time frame, $E_{t x}$ is the transmitted energy of the signal and $d_{m} \in\{ \pm 1\},(m=1,2)$ is the amplitude of pulse $w_{n}^{(k)}(t), n$ represents the order of the orthogonal pulse. The pulses have finite energy and are normalized to ensure equal energy per transmission, that is $\int_{-\infty}^{+\infty}\left|w_{n}(t)\right|^{2} d t=1$ where $0 \leq n \leq M-1$. The pulse repetition interval $T_{f}$ is divided into $N_{h}$ time slots of length $T_{c}$, where $N_{h} T_{c} \leq T_{f},\left\{c_{j}^{(k)}\right\}$ is a pseudorandom TH code sequence, and $0 \leq c_{j}^{(k)}<N_{h}-1 . \delta_{l}^{(k)}$ is the additional time shift from nominal position. For PSM scheme, $n=0,1, \cdots, M-1, d_{m}=1$ and $\delta_{l}^{(k)}=0$. For BPSM scheme, $n=0,1, \cdots, M / 2-1, d_{m}= \pm 1 \quad$ and $\delta_{l}^{(k)}=0$. For OPPM-PSM scheme, $n=0,1, \cdots, N$, $d_{m}=1$ and $\delta_{1}<\delta_{2}<\cdots<\delta_{L}<T_{f}$ where $\delta_{1}=0$. For OPPM-BPSM scheme, $d_{m}= \pm 1$ and other terms are same as OPPM-PSM.

\section{Power Spectral Density Analysis}

In orthogonal pulse based signal, different symbols are transmitted by different order orthogonal pulses. The continuous spectrum, energy spectral density (ESD), changes with symbol. The discrete spectral component changes with orthogonality of the pulses and TH code. Therefore, a mathematical frame work is essential to understand the orthogonal pulse based PSD in the presence of deterministic $\mathrm{TH}$ code. We assume that the analysis is only for 1 user. For simplicity, the superscript/subscript terms in (1) are omitted/modified. After some modification, sum of $M$ symbol can be written from (1) as

$$
s_{p}(t)=\sum_{l=0}^{M-1} \sum_{h=0}^{N_{s}-1} a_{l} w_{l}\left(t-l N_{p} T_{f}+h T_{f}-c_{l, h} T_{c}-\delta_{l}\right)
$$

where $a_{l}$ is the amplitude and $\delta_{l}$ is the pulse position. The terms $a_{l}, \delta_{l}$ and $w_{l}$ are independent and stationary process. The index $p$ is related to $\mathrm{TH}$ code, $c_{l, h}$, and TH period, $N_{p}$. To simplify the analysis of the PSD of TH-UWB signal, it is assumed that the number of time frames for a symbol is $N_{s}$ and it is equal to $N_{p}$. Since (2) depends on the time dithering, it can be written in continuous form as

$$
y(t)=\sum_{l} S_{p}\left(t-l N_{p} T_{f}\right) .
$$

The PSD is computed by evaluating the Fourier transform (FT) of the autocorrelation function of $y(t)$, i.e.,

$$
P_{y}(f)=\mathcal{F}\{E\{y(t) y(t+\tau)\}\}
$$

where $\mathcal{F}\{$.$\} denotes the FT and E\{$.$\} denotes the$ expectation operator. Therefore, the PSD can be expressed as [11].

$$
\begin{aligned}
P_{y}(f)= & \frac{1}{N_{p} T_{f}}\left[E\left\{\left|S_{p}(f)\right|^{2}\right\}-E\left\{S_{p}(f) S_{q}^{*}(f)\right\}\right] \\
& +\frac{1}{\left(N_{p} T_{f}\right)^{2}} \sum_{k} E\left\{S_{p}(f) S_{q}^{*}(f)\right\} \delta\left(f-\frac{k}{N_{p} T_{f}}\right)
\end{aligned}
$$

where $p$ and $q$ are two independent random variables with the same probability distribution function. $S_{p}(f)$ is the FT of $s_{p}(t)$. It can be expressed as

$$
S_{p}(f)=\sum_{l=0}^{M-1} W_{l}(f) T_{l}(f) a_{l} e^{-j 2 \pi f \delta_{l}}
$$

where $W_{l}(f)$ is the FT of the transmitted pulse $w_{l}(t)$. The time domain representation of $(l+2)$ th order MHPs can be expressed as

$$
n w_{l+2}(t)=2 t w_{l+1}(t)-2(l+1) w_{l}(t)
$$

The FT of $w_{l+1}(f)$ can be expressed as

$$
W_{l+1}(f)=j\left[\frac{1}{4 \pi} \dot{W}_{l}(f)-2 \pi f W_{l}(f)\right]
$$

where “ . " stands for derivative with respect to fre- 
quency. For MHP, $W_{0}(f)$ is defined as

$$
W_{0}(f)=2 \sqrt{\pi} e^{-4 \pi^{2} f^{2}}
$$

The time and frequency domain representation of MHPs are given in Figure 1.

$T_{l}(f)$ is the FT of the TH code which transmits the lth symbol

$$
T_{l}(f)=\sum_{h=0}^{N_{S}-1} e^{-j 2 \pi f\left(c_{l, h} T_{c}+\left(l N_{p}+h\right) T_{f}\right)} .
$$

To find the closed form expression of $P_{y}(f)$ in (5), the expectation of $\left|S_{p}(f)\right|^{2}$ is to be evaluated. It is given as

$$
\begin{aligned}
E\left\{\left|S_{p}(f)\right|^{2}\right\}= & E\left\{\sum_{l=0}^{M-1 M-1} \sum_{n=0} W_{l}(f) W_{n}(f)^{*} T_{l}(f)\right. \\
& \left.\times T_{n}(f)^{*} a_{l} a_{n} e^{-j 2 \pi f\left(\delta_{l}-\delta_{n}\right)}\right\} .
\end{aligned}
$$

Since $a_{l}$ and $a_{n}$ are independent random variables derived from the same process and $\delta_{l}$ and $\delta_{n}$ are independent random variables derived from different processes. Therefore, (11) can be rewritten as

$$
\begin{aligned}
E\left\{\left|S_{p}(f)\right|^{2}\right\}= & \sum_{l=0}^{M-1}\left\{\left|W_{l}(f)\right|^{2}\left|T_{l}(f)\right|^{2} E\left\{a_{l}^{2}\right\}+\right. \\
& \sum_{\substack{n=0 \\
n \neq l}}^{M-1} W_{l}(f) W_{n}^{*}(f) T_{l}(f) T_{n}^{*}(f) \\
& \left.\times E\left\{a_{l}\right\} E\left\{a_{n}\right\} E\left\{e^{-j 2 \pi f\left(\delta_{l}-\delta_{n}\right)}\right\}\right\} .
\end{aligned}
$$

Similarly, the second expectation in (5) can be expressed as
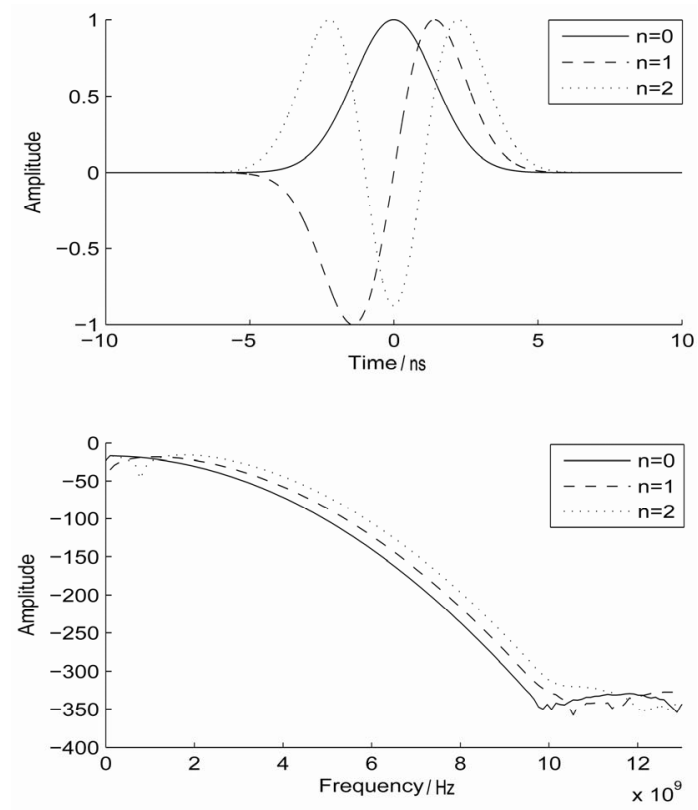

Figure 1. Time and frequency (logarithmic plot) domains representation of modified Hermite pulses (MHPs).

$$
\begin{aligned}
E\left\{S_{p}(f) S_{q}^{*}(f)\right\}= & \sum_{l=0}^{M-1 M-1} \sum_{n=0} W_{l}(f) W_{n}^{*}(f) T_{l}(f) T_{n}^{*}(f) \\
& \times E\left\{a_{l}\right\} E\left\{a_{n}\right\} E\left\{e^{-j 2 \pi f\left(\delta_{l}-\delta_{n}\right)}\right\} .
\end{aligned}
$$

The waveforms $s_{p}(t)$ and $s_{q}(t)$ are generated by two i.i.d processes. Therefore, the expectation in (13) is independent of $l$ and $n$ and equal to the case $l \neq n$ of (12), i.e.,

$$
\begin{aligned}
E\left\{S_{p}(f) S_{q}^{*}(f)\right\} & =E\left\{a_{l}\right\} E\left\{a_{n}\right\} E\left\{e^{-j 2 \pi f\left(\delta_{l}-\delta_{n}\right)}\right\} \\
& \times \sum_{l=0}^{M-1 M-1} \sum_{n=0} W_{l}(f) W_{n}^{*}(f) T_{l}(f) T_{n}^{*}(f)
\end{aligned}
$$

Substituting (12) and (14) in (5), the final PSD can be formulated as in (15).

$$
\begin{aligned}
P_{y}(f)= & E\left\{a_{l}^{2}\right\}-E\left\{a_{l}\right\} E\left\{a_{n}\right\} E\left\{e^{-j 2 \pi f\left(\delta_{l}-\delta_{n}\right)}\right\} N_{p} T_{f} \\
& \sum_{l=0}^{M-1}\left|W_{l}(f)\right|^{2}\left|T_{l}(f)\right|^{2} \\
& +E\left\{a_{l}\right\} E\left\{a_{n}\right\} E\left\{e^{-j 2 \pi f\left(\delta_{l}-\delta_{n}\right)}\right\}\left(N_{p} T_{f}\right)^{2} \\
& \sum_{l=0}^{M-1} \sum_{n=0}^{M-1} W_{l}(f) W_{n}^{*}(f) T_{l}(f) T_{n}^{*}(f) \sum_{k} \delta\left(f-k N_{p} T_{f}\right)
\end{aligned}
$$

\section{Characterization of PSD of TH-UWB Signal}

Although UWB signals are alike in the frequency domain, they are diverse in the time domain due to their different characteristics of time domain parameters $N_{p}$, $T_{f}, a_{l}$ and $w_{l}$. We see that the PSD of orthogonal pulse-based modulation signals consists of continuous and discrete spectral components which change with the order of pulse waveforms and modulation schemes. The variations of PSD over different orthogonal pulse-based signaling are given in the following subsection.

\subsection{PSD of $M$-ary Pulse Shape Modulation (PSM)}

In PSM scheme, symbols are modulated only by the order of orthogonal pulses. The generalized terms in (15) are specified by $a_{l}=1$ and $\delta_{l}=0$. The expectations of these variables are $E\left\{a_{l}^{2}\right\}=1, E\left\{a_{l}\right\} E\left\{a_{n}\right\}_{l \neq n}=0$ and $E\left\{e^{-j 2 \pi f\left(\delta_{l}-\delta_{n}\right)}\right\}=1$ respectively. The PSD of the PSM signal can be written from (15) as

$$
P_{y}(f)=p(f)+p_{k}(f)
$$

where

$$
p(f)=1 N_{p} T_{f} \sum_{l=0}^{M-1}\left|W_{l}(f)\right|^{2}\left|T_{l}(f)\right|^{2}
$$

and 


$$
\begin{aligned}
p_{k}(f)= & 1\left(N_{p} T_{f}\right)^{2} \sum_{l=0}^{M-1 M-1} \sum_{n=0} W_{l}(f) W_{n}^{*}(f) T_{l}(f) T_{n}^{*}(f) \\
& \times \sum_{k} \delta\left(f-k N_{p} T_{f}\right)
\end{aligned}
$$

We see that $p(f)$ is continuous spectrum component. It depends on the TH code and the ESD of the lth order orthogonal pulse. Since ESD of different order orthogonal pulses are not identical, the selection of order of the orthogonal pulses plays an important role for continuous spectral component.

$p_{k}(f)$ is the discrete spectral components which induces UWB interference on the other narrow band systems [12]. The discrete components of the signal appear based on the term $\sum_{k} \delta\left(f-k N_{p} T_{f}\right)$. It shows that the position of discrete component depends on the TH code and its dynamic range of amplitude depends on the orthogonality of pulses. Since pulses are orthogonal in time and frequency domains, the value of $W_{l}(f) W_{n}^{*}(f)$ is approximately zero, as a result, the dynamic range of amplitude of the discrete spectral components becomes very small. This small dynamic range increases the average transmitted power in pulse and improves the UWB system performance. It helps UWB signal to coexist with other systems without any serious performance degradation. In addition, it facilitates UWB signal to keep its spectrum under the FCC spectral mask without minimizing the average transmitted power in the signal.

\subsection{PSD of $M$-ary Biorthogonal PSM (BPSM)}

In BPSM scheme, symbols are modulated by order and amplitude of the pulses, i.e., $a_{l} \in\{ \pm 1\}$ and $\delta_{l}=0$. The expectation of these variables are $E\left\{a_{l}^{2}\right\}=1$,

$$
E\left\{a_{l}\right\} E\left\{a_{n}\right\}_{l \neq n}=0 \text { and } E\left\{e^{-j 2 \pi f\left(\delta_{l}-\delta_{n}\right)}\right\}=1 .
$$

The corresponding PSD of BPSM scheme can be expressed from (15) as

$$
\begin{aligned}
P_{y}(f)= & 1 N_{p} T_{f} \sum_{l=0}^{M-1} \sum_{h=0}^{N_{s}-1 N_{s}-1} \sum_{k=0}\left|W_{l}(f)\right|^{2} \\
& \times \exp \left(-j 2 \pi f\left(\left(c_{l, h}-c_{l, k}\right) T_{c}+(h-k) T_{f}\right)\right)
\end{aligned}
$$

The continuous PSD component of BPSM signal is same as PSM scheme. However, the discrete spectral components become zero due to the antipodal pulse. The PSD of the TH-UWB signal for BPSM scheme is smoothed. This allows the signal to coexist with other NB signals. The extensive studies found that any antipodal signal has only continuous spectral component [12]. The continuous component can be easily fitted to FCC by using appropriate MHPs.

\subsection{PSD of $M$-ary OPPM-BPSM}

For OPPM-BPSM scheme, $a_{l} \in\{ \pm 1\}$ and $\delta_{l}=(l-1) \delta$, where $\delta$ is the constant time shift length. This implies, $E\left\{a_{l}^{2}\right\}=1, E\left\{a_{l} a_{n}\right\}=0$ and

$$
E\left\{e^{-j 2 \pi f m t_{\Delta} \delta}\right\}=\left(1+\cos \left(2 \pi m f T_{\Delta}\right)\right) / 2 .
$$

The corresponding PSD of OPPM-BPSM signal can be expressed as

$$
\begin{aligned}
P_{y}(f)= & 1 N_{p} T_{f} \sum_{l=0}^{M-1} \sum_{h=0}^{N_{s}-1 N_{s}-1} \sum_{k=0}\left|W_{l}(f)\right|^{2} \\
& \times \exp \left(-j 2 \pi f\left(\left(c_{l, h}-c_{l, k}\right) T_{c}+(h-k) T_{f}\right)\right)
\end{aligned}
$$

The PSDs of BPSM and OPPM-BPSM schemes are identical. However, OPPM-BPSM can be used for higher level modulation scheme for higher data rate systems. Therefore, OPPM-BPSM modulation is an attractive choice of TH-UWB signal from several aspects.

\section{Simulation Results and Discussions}

In this section, PSD is provided for orthogonal pulsebased signaling and compared with conventional OPPM scheme. In simulation, different orders of MHPs are used with two different lengths of TH code 8 and 16. The other simulation parameters are set to $T_{f}=60 \mathrm{~ns}$ and pulse width is $7 \mathrm{~ns}$.

Since BPSM and OPPM-BPSM have antipodal signal, they have only continuous spectral component and shape of their spectral is same as continuous component of non antipodal signal. The only difference is that the spectral of antipodal signal does not contain any discrete component. The PSD in non antipodal modulation schemes is more complicated. Since OPPM and OPPM-PSM are special cases of OPPM-BPSM, OPPM and OPPM-PSM have been chosen to compare the PSD of the signal. The PSD of 8-ary OPPM is given in Figure 2 for 3rd order pulse and in Figure 3 for 4 th and 5th order pulses with TH code of length 8 and $T_{c}=7.5$. Since each time only one pulse is used in OPPM scheme, orthogonality is maintained by position not by pulse. The 3rd order pulse almost satisfies the FCC spectral mask except some discrete components. However, 4th and 5th order pulses do not satisfy the FCC spectral mask shown in Figure 3. The dynamic range of the amplitude of discrete components of OPPM scheme is about $8 \mathrm{~dB}$ which is very high. The power of the signal is calculated based on the line where the dynamic range is zero $(4 \mathrm{~dB}$ bellow from the pick point). As FCC rules, pick amplitude must be bellow the $-41.25 \mathrm{dBm}$ limit. Therefore, the power of the signal is calculated based on the line which is maximized up to $-45.25 \mathrm{dBm}$. As a result, signal provides low average transmitted power which degrades the system 


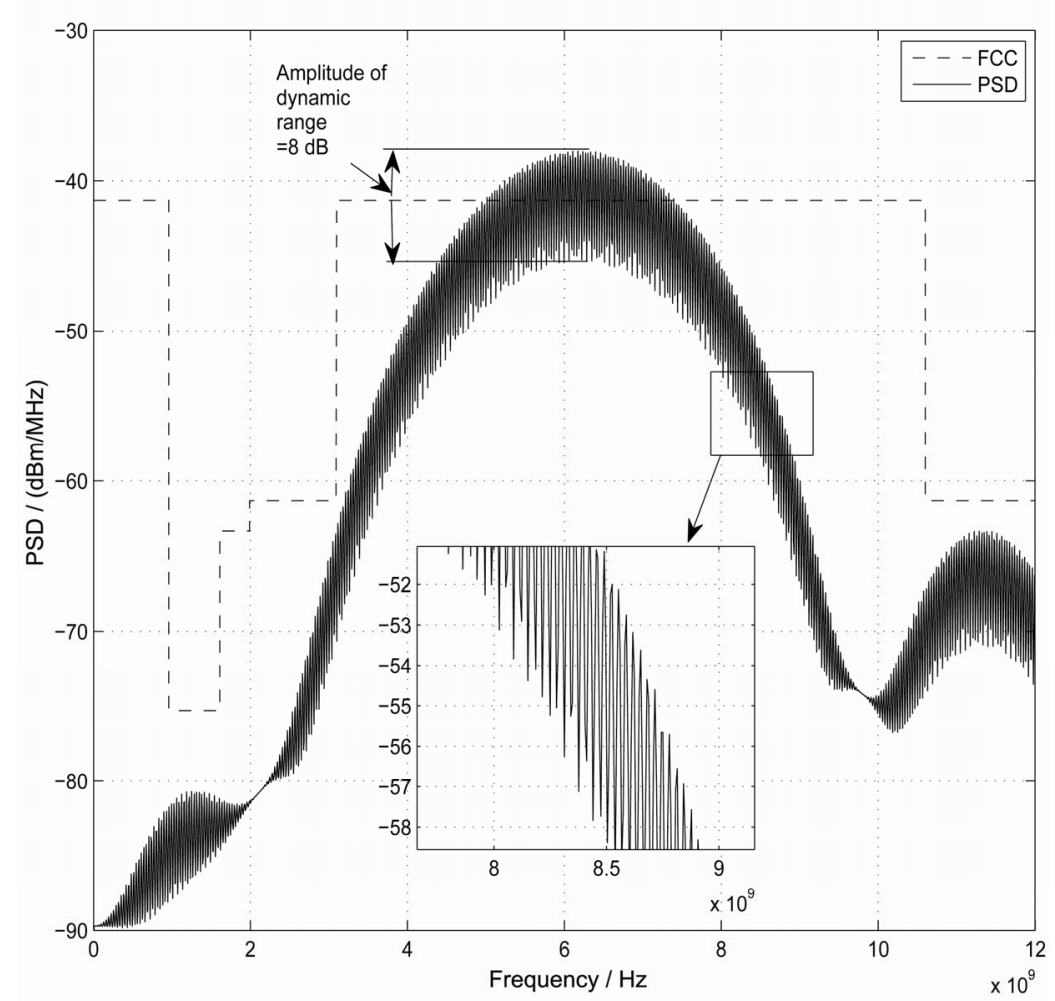

Figure 2. PSD of 8-ary OPPM scheme with 3rd order MHP and TH code length is 8.
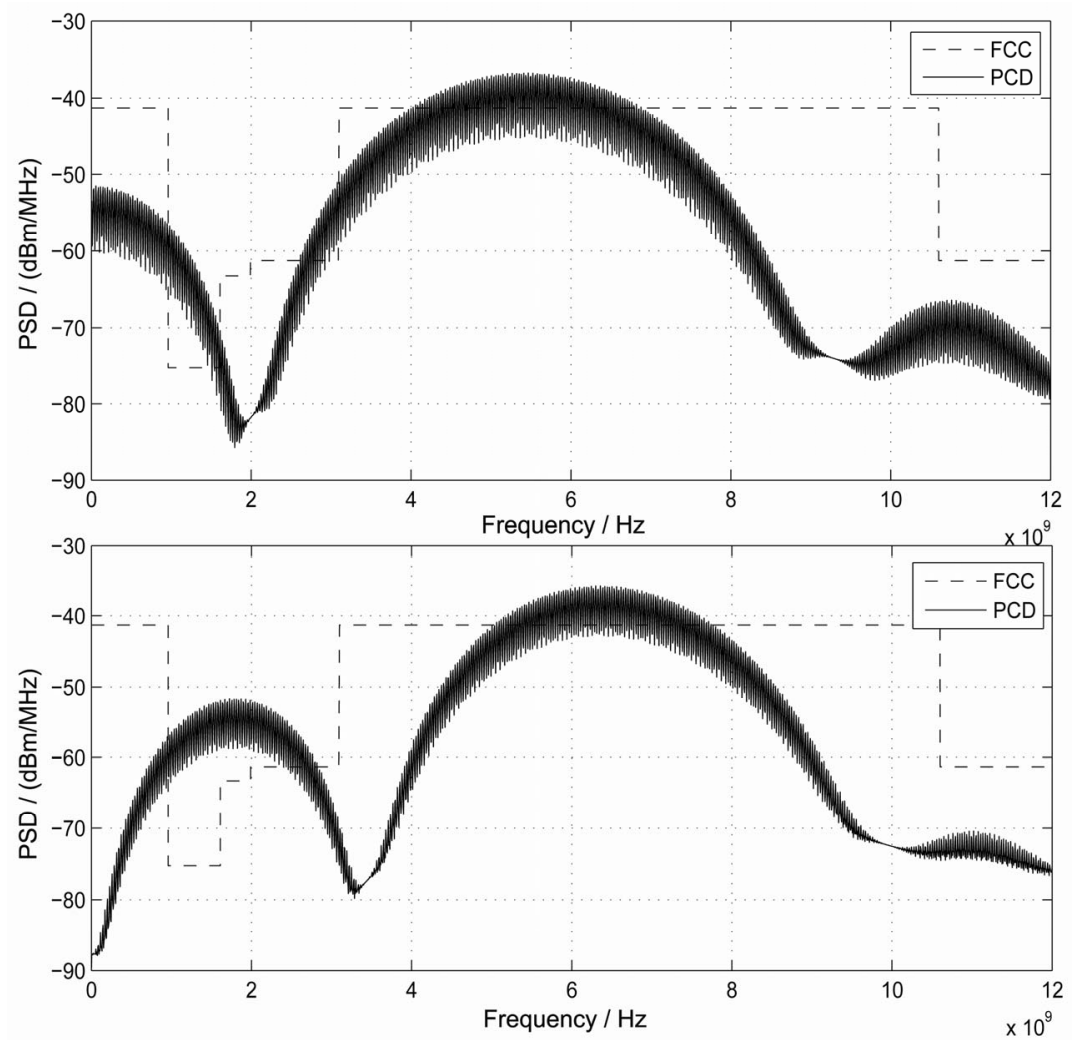

Figure 3. (a) PSD of 8-ary OPPM scheme with 4th order MHP; (b) PSD of 8-ary OPPM scheme with 5th order MHP and TH code length is 8 . 


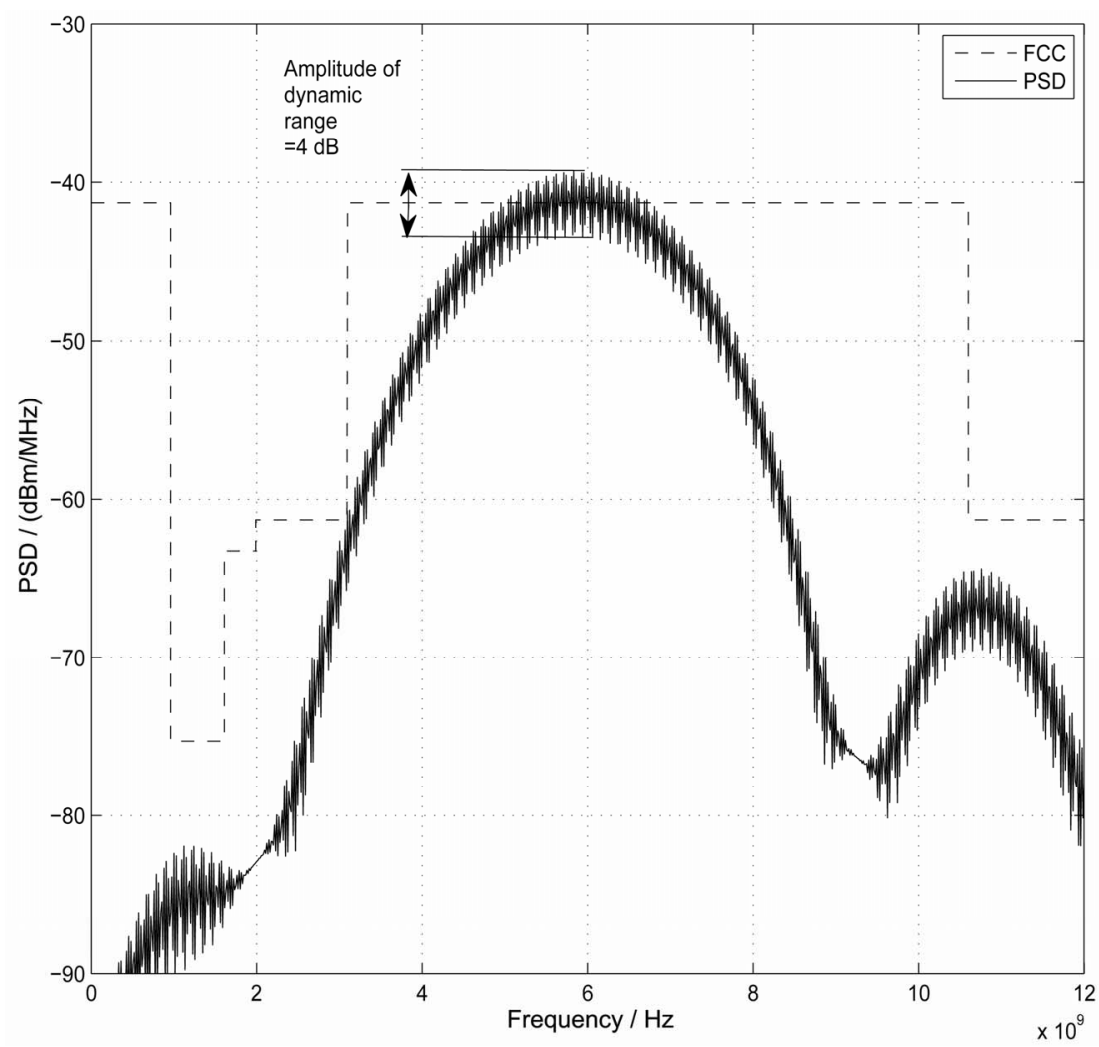

Figure 4. PSD of 8-ary OPPM-PSM schemes for 4 positions and 5 pulses (0th and 3rd) with TH code of length 8.

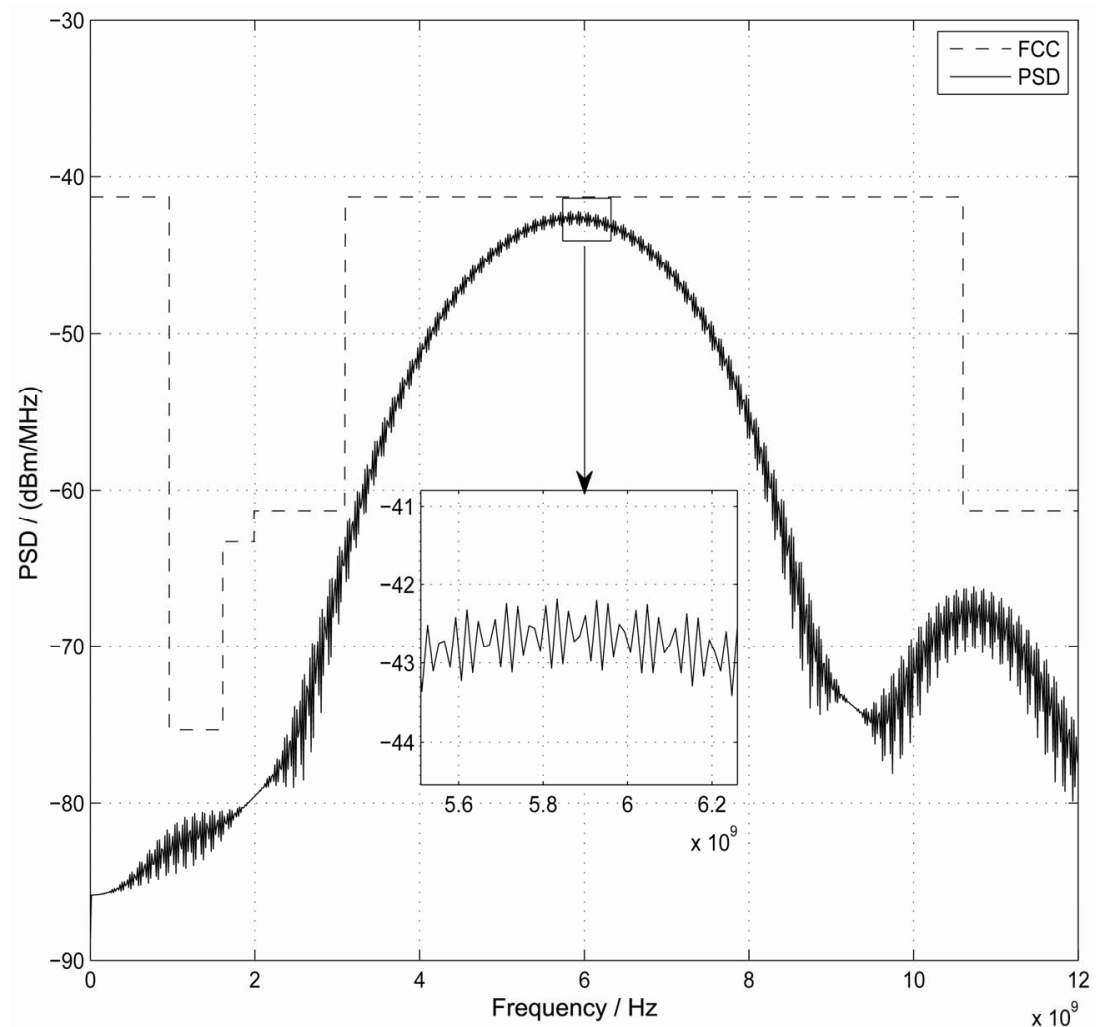

Figure 5. PSD of 8-ary OPPM-PSM schemes for 4 positions and 2 pulses 0th and 3rd with TH code of length 16. 
performance. Not that if the dynamic range becomes zero, the maximum limit becomes $-41.25 \mathrm{dBm}$.

Figure 4 shows the PSD of 8-ary OPPM-PSM for 4 positions and 2 orthogonal pulses with TH code of length 8. We see that that dynamic range of the amplitude of the discrete spectral component of OPPM-PSM scheme is 4 $\mathrm{dB}$ which is lesser than the OPPM scheme even the same length of TH code is used. It is because of the orthogonality of pulses. So by reducing dynamic range, we can improve the UWB system performance by increasing the average transmitted power in the signal pulse as well as we can reduce the UWB interference over other radio systems. Again by applying TH code over these orthogonal pulse-based modulations, dynamic range of amplitude of discrete component further could be reduced. Figure 5 shows the PSD of 8-ary OPPM-PSM with $\mathrm{TH}$ code of length 16 and $T_{c}=3.75$. The dynamic range is almost reduced to $1 \mathrm{~dB}$. However, it can not be reduced to zero whatever the length of TH code used. We also see that the average transmitted power in Figure 5 is more than the previous cases. Therefore, orthogonal pulse-based TH-UWB signaling has several advantages than its complexity burden.

\section{Conclusion}

PSD of orthogonal pulse-based TH-UWB signal has been analyzed based on stochastic signal theory. This shows that the continuous and discrete components change with order of pulses and modulation schemes. The discrete component can be removed by employing an antipodal signal. If the signal is not antipodal, the dynamic range of amplitude of the discrete component can be reduced by using orthogonal pulse-based modulation and long TH code. Therefore, it has been proved that the orthogonal pulse-based signaling not only reduce the dynamic range of discrete spectral component also improve the system performance by increasing the average transmitted power in the signal.

\section{References}

[1] S. Majhi, A. S. Madhukumar and A. B. Premkumar, "Reduction of UWB Interference at NB Systems Based on a Generalized Pulse Waveform," IEICE Electronics Express, Vol. 3, 2006, pp. 361-367.

[2] W. Lee and S. Jitapunkul, "Orthogonal Pulse Design for UWB Systems with Timing Jitter,” International Con- ference on Advanced Communication Technology, Phoenix, February 2009, pp. 22-27.

[3] A. B. P. Sudhan Majhi, A. S. Madhukumar and P. Richardson, "Combining OOK with PSM Modulation for TH-UWB Radio Systems: A Performance Analysis," EURASIP Journal on Wireless Communications and Networking, Vol. 2008, 2008.

[4] C. Michell, G. T. F. de Abreu and R. Kohno, “Combined Pulse Shape and Pulse Position Modulation for High Data Rate Transmission in Ultra-Wideband Communication," International Journal of Wireless Information Networks, Vol. 10, October 2003, pp. 167-178.

[5] S. Majhi, A. S. Madhukumar, A. B. Premkumar and F. Chin, "M-ary Signaling for Ultra Wideband Communication Systems Based on Pulse Position and Orthogonal Pulse Shape Modulation,” IEEE Wireless Communication and Networking Conference (WCNC), March 2007, pp. 2795-2799.

[6] I. Dotlic and R. Kohno, "Design of the Family of Orthogonal and Spectrally Efficient Uwb Waveforms," IEEE Journal of Selected Topic in Signal Processing, Vol. 1, No. 1, June 2007, pp.21-30.

[7] Y. Chen, E. Gunawan, K. S. Low, S. Wang, Y. Kim and C. B. Soh, "Pulse Design for Time Reversal Method as Applied to Ultrawideband Microwave Breast Cancer Detection: A Two-Dimensional Analysis," IEEE Transactions on Antennas and Propagation, Vol. 55, No.1, January 2007, pp. 194-204.

[8] Y. Kim and F. B. Womack, "Performance Evaluation of UWB Systems Exploiting Orthonormal Pulses," IEEE Transactions on Communications, Vol. 55, No. 5, May 2007, pp. 929-935.

[9] J. H. Hwang, S. C. Kim, B. K. S. Yoon and J. S. Park, "Performance Analysis of PO-THMA UWB System Using Mutually Orthogonal MHP Pulses,” IEEE Transactions on Consumer Electronics, Vol. 53, No. 1, February 2007. pp. 39-45.

[10] J. A. N. da Silva and M. L. R. de Campos, "Spectrally Efficient UWB Pulse Shaping with Application in Orthogonal PSM," IEEE Transactions on Communications, Vol. 55, No. 2, 2007, pp. 313-322.

[11] J. E. Padgett, J. C. Koshy and A. A. Triolo, "Physical-Layer Modeling of UWB Interference,” White Paper of Telcordia Technologies, January 2003, pp. 1-121.

[12] S. Majhi, A. S. Madhukumar and Z. Ye, "Coexisting Narrowband and Ultra Wideband Systems: Analysis of Power Spectral Density and In-Band Interference Power," WSEAS Transaction on Communication, Vol. 6, February 2007, pp. 118-124. 